\title{
TTAS a new stilbene derivative that induces apoptosis in Leishmania infantum
}

\author{
Manlio Tolomeo ${ }^{\mathrm{a}, *}$, Marinella Roberti ${ }^{\mathrm{b}}$, Leonardo Scapozza ${ }^{\mathrm{c}}$, Chiara Tarantelli ${ }^{\mathrm{d}}$, Elisa Giacomini ${ }^{\mathrm{b}}$, \\ Lucina Titone $^{\mathrm{a}}$, Laura Saporito ${ }^{\mathrm{a}}$, Paola Di Carlo ${ }^{\mathrm{a}}$, Claudia Colomba ${ }^{\mathrm{a}}$ \\ ${ }^{a}$ Department of Health Promotion Sciences, Section of Infectious Diseases, University of Palermo, Via del Vespro 129, 90127 Palermo, Italy \\ ${ }^{\mathrm{b}}$ Department of Pharmaceutical Sciences, University of Bologna, Via Belmeloro 6, 40126 Bologna, Italy \\ ${ }^{\mathrm{c}}$ Pharmaceutical Biochemistry Group, School of Pharmaceutical Sciences, University of Geneva, Quai Ernest-Ansermet 30, 1211 Genève 4, Switzerland \\ dDepartment of Oncology and Experimental Medicine, University "G. d'Annunzio" Chieti-Pescara, Chieti, Italy
}

\section{H I G H L I G H T S}

- We evaluated the Leishmanicidal activity of a pool of new stilbene derivatives.

- Trans-3,4',5-trimethoxy-3'-aminostilbene (TTAS) is the most active stilbene.

- TTAS induces apoptosis in Leishmania parasites.

- Leishmania tubulin is the putative target of TTAS.

\section{A R T I C L E I N F O}

\section{Article history:}

Received 15 March 2012

Received in revised form 6 October 2012

Accepted 11 October 2012

Available online 26 October 2012

\section{Keywords:}

Leishmaniasis

Programmed cell death

Stilbenes

Tubulin

\section{G R A P H I C A L A B S T R A C T}

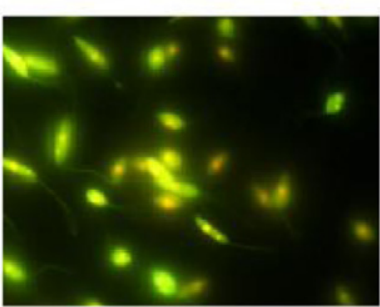

Living Leishmania parasites

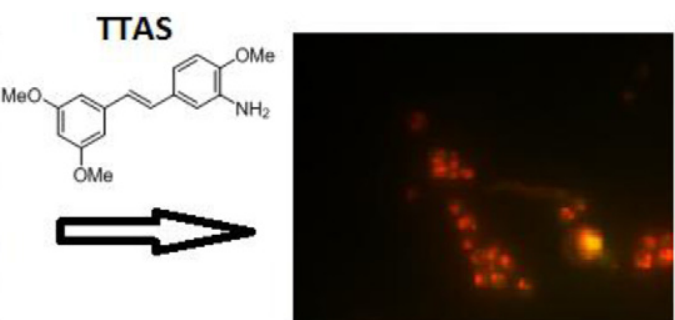

Apoptotic Leishmania parasites

\begin{abstract}
A B S T R A C T
Leishmania parasites are able to undergo apoptosis (programmed cell death), similarly to mammalian cells. Recently it was demonstrated in vitro the anti-leishmanial effect of some natural and synthetic stilbenoids including resveratrol and piceatannol. In this study we evaluated the Leishmanicidal activity of a pool of stilbene derivatives which had previously shown high apoptotic efficacy against neoplastic cells. All the compounds tested were capable to decrease the parasite viability in a dose-dependent manner. Trans-stilbenes proved to be markedly more effective than cis-isomers. This was different from that observed in tumor cells in which cis-stilbenes were more potent cytotoxic agents. Trans-3,4',5-trimethoxy-3'-amino-stilbene (TTAS) was the most active stilbene showing in Leishmania infantum a $\mathrm{LD}_{50}$ value of $2.6 \mu \mathrm{g} / \mathrm{mL}$. In contrast TTAS showed a low toxicity when tested on normal hemopoietic cells. This compound induced apoptosis in parasites by disrupting the mitochondrial membrane potential. Moreover it shows the ability to block Leishmania parasites in $\mathrm{G}_{2}-\mathrm{M}$ phase of cell cycle in agreement with the data obtained by affinity chromatography that identify tubulin as the putative target of TTAS. In conclusion, our results indicate that some stilbene derivatives are highly effective as anti-leishmanial agents and TTAS represents a pro-apoptotic agent in Leishmania parasites that merit further in vivo investigation.
\end{abstract}

(c) 2012 Elsevier Inc. All rights reserved.

\section{Introduction}

Leishmaniasis are globally widespread parasitic diseases caused by several protozoan parasites of the Leishmania genus. They are

\footnotetext{
* Corresponding author. Address: Department of Health Promotion Sciences Section of Infectious Diseases, University of Palermo, Interdepartmental Research Centre on Clinical Oncology (C.I.R.O.C.), University of Palermo, Via del Vespro 129, 90127 Palermo, Italy. Fax: +39 0916554059

E-mail address: mtolomeo@hotmail.com (M. Tolomeo).
}

transmitted to humans by the bite of an insect vector, generally the females sandfly of the genus Phlebotomus in the Old World, or Luztomiya in the New World. Infections can vary from simple cutaneous leishmaniasis (CL) to mucocutaneous (MCL) and visceral leishmaniasis (VL) known as kala-azar, which is fatal if not treated. It is estimated that about half a million people die annually with VL and over 350 million people live at risk of this infection. Moreover, AIDS and other immunosuppressive conditions enhanced the risk of Leishmania-HIV co-infection and contribute to the appearance of new severe clinical forms of the disease. To date, the greatest 
prevalence of Leishmania-HIV co-infection has been in the Mediterranean basin (Cruz et al., 2006; Colomba et al., 2009). Current leishmaniasis treatment is based on chemotherapy, which relies on a handful of drugs with serious limitations such as high cost and toxicity, difficult route of administration, and lack of efficacy in endemic areas (Murray et al., 2005; Croft et al., 2006; Baiocco et al., 2009; Palumbo, 2009). Extensive evidence from studies in animal models indicates that good protection can be achieved by immunization, however, to date no vaccine is available and also for this reason there is an urgent need to develop safer, cheaper and more effective antileishmanial agents.

Natural products are potentially rich sources of novel active molecules that may serve as lead or structural template for drug discovery. In this context, in the last years several natural compounds and their synthetic analogs have been tested against Leishmania, and many of them have demonstrated potential as leishmanicidal agents (Changtam et al., 2010; Tipparaju et al., 2008; Marin et al., 2009; Mishra et al., 2009a,b).

Stilbene-based compounds are widely represented in nature (Shen et al., 2009), and have become of particular interest to chemists and biologists because of their wide range of biological effects including chemopreventive, antitumor, antioxidant, antimicrobial, anti-inflammatory and antihistaminic activities (Hart, 1981; Fremont, 2000; Young and Chaplin, 2004). Recently, several natural and synthetic stilbenoids have been studied for their leishmanicidal properties (del Rey et al., 1999; del Olmo et al., 2001; Getti et al., 2006), and some of them, including resveratrol (trans-3,4',5-trihydroxystilbene) (Kedzierski et al., 2007) and piceatannol (trans3,3', $4^{\prime}, 5$-tetrahydroxystilbene) (Duarte et al., 2008), have shown anti-leishmanial activity in vitro.

In previous works, we have described the synthesis of a small library of cis- and trans-resveratrol based derivatives with the aim of discovering new lead compounds with anticancer potential (Roberti et al., 2003). The best results were obtained with cis-3,4',5-trimethoxy-3'-hydroxy-stilbene $\mathbf{1}$ and its 3'-amino derivative $\mathbf{2}$, which showed pro-apoptotic activity at nanomolar concentrations in HL60 leukemia cells. We have also demonstrated that pterostilbene $\mathbf{3}$ and 3'-hydroxy pterostilbene $\mathbf{4}$ possess interesting antileukemic properties and may constitute effective and powerful drugs in MDR and apoptosis-resistant hematological malignancies. Notably, the toxicity of these compounds, evaluated on normal hemopoietic cells (CFU-GM), was very low (Tolomeo et al., 2005; Cao et al., 2008).

In light of the above, here we present a study in which we evaluated the anti-leishmanial activity of stilbenes 1-2,4 togheter with their correspondent isomers 5-7 (1 vs 5, 2 vs 6, 4 vs 7, Table 1). Moreover we tested the activity of pterostilbene $\mathbf{3}$.

Compound 6, namely trans-3,4',5-trimethoxy-3'-amino-stilbene (TTAS), was the most active derivative showing a $\mathrm{LD}_{50}$ value of $2.6 \mu \mathrm{g} / \mathrm{mL}$. This finding prompted us to address the questions regarding the cellular target and the molecular mechanism underlying the observed toxicity toward the parasite. To this end we carried out a chemical proteomic analysis that led us to the identification of tubulin as a putative target of TTAS. Moreover, from the loss in mitochondrial membrane potential in Leishmania parasite, we hypothesize the activation of mitochondrial apoptotic pathway.

\section{Materials and methods}

\subsection{Promastigote culture and cell treatments}

The Leishmania infantum strain MON1/IPT1 promastigotes were cultured in blood agar as previously described (Sengupta et al., 1999). Briefly, the cells were routinely maintained on solid blood agar slants containing $1 \%$ glucose, $5.2 \%$ brain heart infusion agar extract and rabbit blood $(6 \% \mathrm{v} / \mathrm{v})$ with gentamycin at a final con- centration of $1-1.5 \mathrm{mg} \mathrm{mL}^{-1}$ of medium at $25^{\circ} \mathrm{C}$. Axenic amastigotes were obtained from promastigote cultures of Leishmania infantum strain MON1/IPT1. Promastigotes growing in log phase were harvested, washed in PBS, adjusted to a concentration of $10^{6}$ parasites $/ \mathrm{mL}$ in fresh medium at $\mathrm{pH} 5$ and incubated at $32^{\circ} \mathrm{C}$. Complete transformation into amastigotes was achieved within 10 days of incubation. For experimental purposes, exponentially growing cells were collected and resuspended in RPMI medium supplemented with $10 \%$ fetal calf serum to achieve a culture density of $10^{6}$ cells $/ \mathrm{mL}$. Cells were dispensed in 24-well culture plates and appropriate concentrations of each compound were added.

\subsection{Evaluation of live and dead parasites}

To evaluate the leishmanicidal activity of stilbene-based compounds the percentage of live and dead parasites was determined morphologically after labeling with acridine orange $(100 \mu \mathrm{g} / \mathrm{mL})$ and ethidium bromide $(100 \mu \mathrm{g} / \mathrm{mL})$. After $48 \mathrm{~h}$ exposure to each compound, parasites $\left(1 \times 10^{6}\right)$ were centrifuged and the pellet was resuspended in $25 \mu \mathrm{L}$ of the dye mixture. Ten $\mu \mathrm{L}$ of the mixture was examined in oil immersion with a $100 \times$ objective using a fluorescence microscope Nikon Eclipse E200. Live parasites were determined by the uptake of acridine orange (green fluorescence) and exclusion of ethidium bromide (red fluorescence). Dead parasites was determined by the uptake of ethidium bromide (red fluorescence). The percentage of parasite viability was determined after counting at least 300 parasites.

\subsection{Clonal assays}

To evaluate the cytotoxic effects of stilbenes on normal hemopoietic progenitor cells, a clonal assay for CFU-GM (colony-forming units-granulocyte macrophage) was performed. Bone marrow mononucleated cells were obtained from bone marrow aspirates of five normal volunteers. Bone marrow (3-5 mL) was diluted in RPMI 1640, layered over a Ficoll-Hypaque gradient (density, 1.077), centrifuged at $400 \mathrm{~g}$ for $30 \mathrm{~min}$, and the interface mononuclear cells were collected. The interface cells were washed 3 times in PBS, counted, and resuspended at a concentration of $1 \times 10^{5}$ in MEM containing $0.9 \%$ methylcellulose, $30 \% \mathrm{FCS}, 10^{-4} \mathrm{M}$ betamercaptoethanol, $5 \%$ medium conditioned by leukocytes in the presence of phytohemagglutinin (PHA-LCM) in $15 \mathrm{~mm}$ plastic dishes. After 7 days of culture at $37^{\circ} \mathrm{C}$ in an environment of $5 \%$ $\mathrm{CO}_{2}$ and $100 \%$ humidity, the number of CFU-GM, was evaluated.

\subsection{Flow cytometry analysis of cell cycle}

Parasites were incubated for $24 \mathrm{~h}$ with the stilbene-based compounds at $26^{\circ} \mathrm{C}$. Afterward, the parasites were washed 3 times with PBS containing 0.02 M EDTA to avoid clumps and were then fixed with cold methanol for $24 \mathrm{~h}$. The parasites were resuspended in $0.5 \mathrm{~mL}$ of PBS containing RNase I $(50 \mu \mathrm{g} / \mathrm{mL})$ and PI $(25 \mu \mathrm{g} / \mathrm{mL})$ and were then incubated at $25^{\circ} \mathrm{C}$ for $20 \mathrm{~min}$. The material was kept on ice until analysis. The stained parasites were analyzed in a single-parameter frequency histograms by using a FACScan flow cytometer (Becton Dickinson, San Jose, CA).

\subsection{Determination of apoptosis by Annexin $V$}

Externalization of phosphatidylserine on the outer membrane of parasites with and without treatment was determined by using Annexin V labeling kit following the manufacturer's protocol (Annexin-V-FITC Apoptosis Detection Kit Alexis, Lausen, Switzerland) (Singh et al., 2005). Briefly, parasites $\left(2 \times 10^{6}\right)$ were washed with PBS and centrifuged at $500 \mathrm{~g}$ for $5 \mathrm{~min}$. The pellet was suspended in $100 \mu \mathrm{L}$ of staining solution containing FITC-conjugated Annex- 
Table 1

$\mathrm{LD}_{50}$ values $(\mu \mathrm{g} / \mathrm{mL})$ of stilbenes 1-7 against the Leishmania infantum strain IPT1.

\begin{tabular}{|c|c|c|c|}
\hline Compounds & Structure & Promastigotes $\mathrm{LD}_{50}(\mu \mathrm{g} / \mathrm{mL})$ & Amastigotes $\mathrm{LD}_{50}(\mu \mathrm{g} / \mathrm{mL})$ \\
\hline 1 & $\mathrm{Me}$ & $17.5 \pm 2.3$ & $28.6 \pm 3.4$ \\
\hline 2 & & $18 \pm 1.5$ & $30.2 \pm 4$ \\
\hline 3 & & $7.6 \pm 3$ & $10.3 \pm 1.8$ \\
\hline 4 & & $6.5 \pm 0.8$ & $9.7 \pm 2.1$ \\
\hline 5 & & $10.9 \pm 1.8$ & $22.6 \pm 3.9$ \\
\hline 6 (TTAS) & & $2.6 \pm 0.4$ & $4.3 \pm 0.6$ \\
\hline 7 & $\mathrm{MeO}$ & $16.1 \pm 3.2$ & $28.4 \pm 3.5$ \\
\hline Pentostan & & $2.1 \pm 0.5$ & $8.3 \pm 0.9$ \\
\hline
\end{tabular}

Values represent the mean \pm SE for four independent experiments.

in-V and propidium iodide (Annexin-V-Fluos Staining Kit, Roche Molecular Biochemicals, Mannheim, Germany) and incubated for $15 \mathrm{~min}$ at $20^{\circ} \mathrm{C}$. Annexin $\mathrm{V}$ positive parasites were determined by flow cytometry (Becton Dickinson).

\subsection{Measurement of mitochondrial membrane potential}

Mitochondrial transmembrane potential was investigated using a JC-1 probe. JC-1 aggregates in mitochondria and fluoresces red at higher transmembrane potential but at lower transmembrane potentials it cannot accumulate in the mitochondria and remains as monomers in the cytoplasm that fluoresce green. Briefly, cells were labeled for $10 \mathrm{~min}$ with $10 \mu \mathrm{M} \mathrm{JC}-1$ (BD $^{\mathrm{TM}}$ MitoScreen JC-1 Kit, BD Bioscences Pharmigen, San Diego, CA) at $37^{\circ} \mathrm{C}$ and washed 2 times. Multi-parameter flow cytometry analysis was performed by a Becton Dickinson flow cytometer.

\subsection{Affinity chromatography and identification by mass spectroscopy}

Compound $\mathbf{6}$ was immobilized via the primary amino group on epoxi-activated agarose providing a 12-atoms spacer (1,4-bis(2:3epoxypropoxy)-butane). Swollen and thoroughly washed matrix was resuspended in two volumes of $25-100 \mathrm{mM}$ ligand dissolved in $50 \% \mathrm{DMF} / 50 \mathrm{mM} \mathrm{Na} \mathrm{CO}_{3}, 50 \mathrm{mM} \mathrm{NaCl}, \mathrm{pH} 9.5$. Coupling was performed for $16 \mathrm{~h}$ at $25^{\circ} \mathrm{C}$. After 5 washes with 50\% DMF/ $50 \mathrm{mM} \mathrm{Na}_{2} \mathrm{CO}_{3}, 50 \mathrm{mM} \mathrm{NaCl}, \mathrm{pH} 9.5$, remaining reactive groups were blocked with $1 \mathrm{M}$ ethanolamine ( $\mathrm{pH} 8$ ) and thoroughly washed with low/high pH buffers. A control matrix was prepared without ligand and treated as described above. Direct absorbance scans of the immobilized ligand on the matrix resuspendend in $50 \%$ glycerol solution (v/v) clearly confirmed successful coupling (data not shown). The amount of compound bound to the matrix was determined by back calculation of amount of compound applied and amount recovered by UV determination. Routinely 50$80 \mu \mathrm{mol} / \mathrm{mL}$ compound were bound. The resin was incubated in 2.5 volumes of Leishmania infantum total cell lysate $(12 \mathrm{mg} / \mathrm{mL}$ protein) at $4{ }^{\circ} \mathrm{C}$ for $3 \mathrm{~h}$. After washing with lysis buffer (4 times), the matrix was heated at $95{ }^{\circ} \mathrm{C}$ for 5 min with Laemmli sample buffer, directly loaded and separated by $12 \%$ SDS-PAGE. Resolved proteins were visualized by silver staining. The control matrix was incubated with the same amount of lysate and treated equally. Protein bands were excise from the gels and fragments resulting from trypsin digestion were analyzed by LC/ESI/MS/MS-QTOF mass spectrometry. Data base searches were performed by using the ProteinLynx Global Server (all species) and Mascot (all species) search programs. A score $>48$ indicates identity $(p<0.05)$.

\section{Results}

\subsection{Cytotoxicity}

Compounds 1-7 were tested for leishmanicidal activity against promastigotes and amastigotes of Leishmania infantum (IPT1 strain). Parasites were incubated in presence of different concentrations 
of each compound and viability was evaluated after $48 \mathrm{~h}$, as described in materials and methods. The sample concentration causing $50 \%$ reduction in parasite viability $\left(\mathrm{LD}_{50}\right)$ was used as the parameter for leishmanicidal activity. $\mathrm{LD}_{50}$ values ranged from $18 \mu \mathrm{g} / \mathrm{mL}$ to $2.6 \mu \mathrm{g} / \mathrm{mL}$ in promastigotes and from $30.2 \mu \mathrm{g} / \mathrm{mL}$ to $4.3 \mu \mathrm{g} / \mathrm{mL}$ in amastigotes as depicted in Table 1 . The cis-stilbene derivatives were less active than their corresponding trans isomers (compare 1 vs 5, 2 vs 6, 4 vs 7). The pterostilbene 3 showed an activity comparable to that of its $3^{\prime}$-hydroxy derivative 4 . In the present study the most active compound was trans-3,4',5-trimeth-

(a)

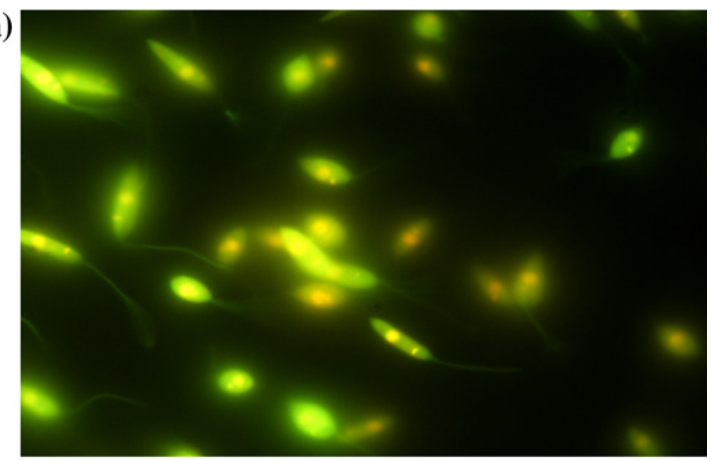

(b)

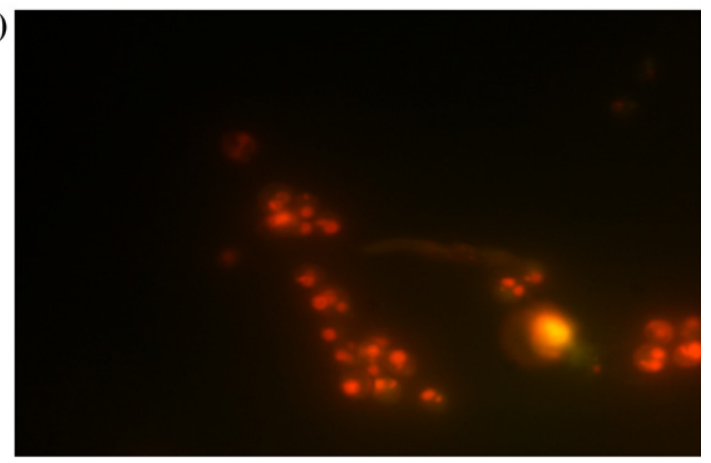

Fig. 1. Morphologic changes observed in Leishmania infantum after exposure to TTAS. Cells were exposed to a mixture of acridine orange and ethidium bromide as described in Section 2. Live parasites were determined by the uptake of acridine orange (green fluorescence) and exclusion of ethidium bromide (red fluorescence). Dead parasites was determined by the uptake of ethidium bromide (red fluorescence). (a) Control. (b) promastigotes exposed to $7 \mu \mathrm{g} / \mathrm{mL}$ TTAS for $48 \mathrm{~h}$; parasites uptake ethidium bromide showing a loss of volume and nuclear condensation. (For interpretation of the references to color in this figure legend, the reader is referred to the web version of this article.)

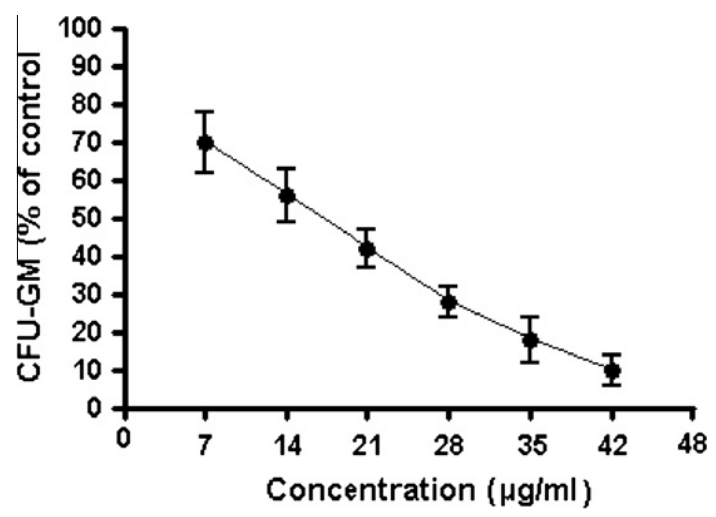

Fig. 2. Effects of compound TTAS on normal CFU-GM. Cells were exposed to different concentrations of TTAS. The number of CFU-GM was calculated after 7 days of cell culture. oxy-3'-amino-stilbene (6, TTAS) that showed a $\mathrm{LD}_{50}$ of $2.6 \mu \mathrm{g} / \mathrm{mL}$ (Table 1). Compared to Pentostan (sodium stibogluconate) used as reference compound, TTAS showed a slightly lower activity in promastigotes but was more active in amastigotes (Table 1).

Parasites exposed $48 \mathrm{~h}$ to TTAS and stained with the acridine orange and ethidium bromide mixture showed changes in morphology (like loss of cell volume and nuclear condensation) that shares many characteristics with metazoans apoptotic death (Fig. 1).

In order to evaluate the toxicity of TTAS on normal hemopoietic cells, bone marrow cells were treated with the compound and the number of CFU-GM was evaluated after 7 days of culture (Fig. 2). TTAS showed an $\mathrm{IC}_{50}$ on normal CFU-GM of $17.7 \mu \mathrm{g} / \mathrm{mL}$, more than 6 times higher than that showed on the Leishmania infantum strain.

\subsection{Cell cycle}

The effect of TTAS on cell cycle distribution was analyzed in Leishmania infantum. Cells were cultured in the presence of $7 \mu \mathrm{g} /$ $\mathrm{mL}$ of TTAS for $24 \mathrm{~h}$. Flow cytometric analysis of cell cycle was carried out after staining of parasites with propidium iodide. As shown in Fig. 3, TTAS induced the arrest of Leishmanias prevalently in $G_{2}-M$ phase of the cell cycle and an increase of the sub- $G_{1}$ apoptotic peak.

\subsection{Determination of apoptosis by Annexin $V$}

The Annexin $\mathrm{V}$ assay provides a simple and effective method to detect apoptosis. This assay takes advantage on the fact that (i) phosphatidylserine (PS) is translocated from the inner (cytoplasmic) leaflet of the plasma membrane to the outer (cell surface) leaflet soon after the induction of apoptosis, (ii) the Annexin V protein has a strong and specific affinity for PS.

As shown in Fig. 4, treatment of parasites with TTAS induces PS externalization (increase of cells in the bottom right quadrant of the flow cytometric dot plot) indicating that the cause of parasite death is the activation of apoptosis.

\subsection{Mitochondrial membrane potential detection}

TTAS induces change in mitochondrial membrane potential in Leishmania infantum strain IPT1. Studies in a variety of blood, hepatic, and immune cell models have shown that a fall in mitochondrial membrane potential is one of the earliest events in apoptosis (Susin et al., 1998). In fact, opening of the mitochondrial permeability transition pore has been demonstrated to induce depolarization of the transmembrane potential, release of apoptogenic factors and loss of oxidative phosphorylation. The loss of mitochondrial membrane potential has been observed to play a key role in drug-induced death in protozoans such as Leishmania (Sen et al., 2004a,b).

In this study, the modifications in mitochondrial membrane potential induced in Leishmania infantum strain IPT1, were evaluated using the fluorescent cationic dye JC-1. The JC- 1 dye accumulates in the mitochondria of healthy cells as aggregates, which are fluorescent red in color. Upon the onset of apoptosis, the mitochondrial potential collapses and the JC- 1 dye can no longer accumulate in the mitochondria and remains in the cytoplasm in a monomeric form which fluoresces green. The differential distribution of the red and green forms of the dye is easily analyzed by flow cytometry. The dye is highly specific and sensitive and gives very little background.

TTAS-induced loss in mitochondrial membrane potential in Leishmania infantum strain IPT1 is demonstrated by the decrease in red fluorescence (FL2) in the cytoplasm (Fig. 5). 

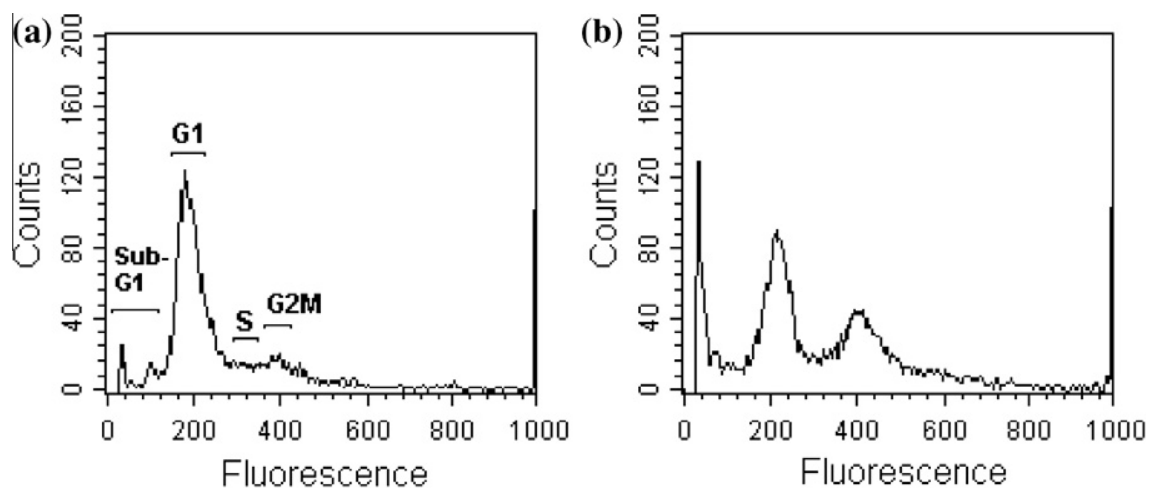

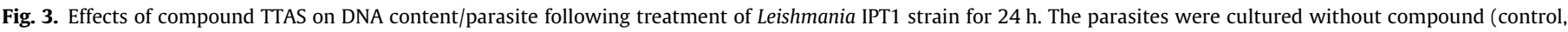

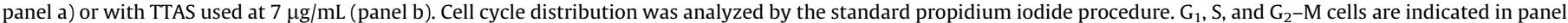
a.
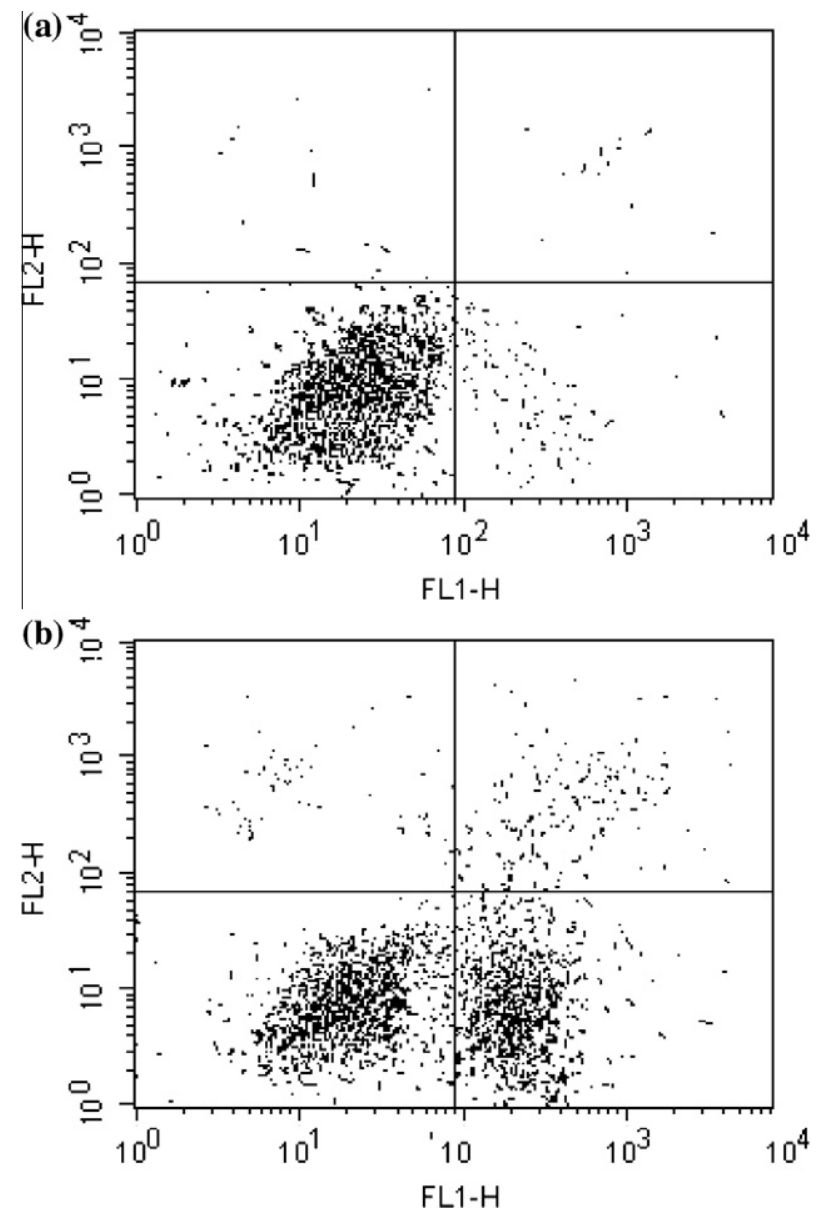

Fig. 4. Apoptosis evaluation by the Annexin-V test. Flow cytometric analysis of apoptotic Leishmanias after $48 \mathrm{~h}$ treatment without (panel a) or with (panel b) TTAS $(7 \mu \mathrm{g} / \mathrm{mL})$. Parasites were stained with Annexin V and propidium iodide. Cells in the bottom right quadrant of each dot plot represent the percentage of cells in early apoptosis (Annexin-V-positive and PI-negative). Data are representative of three separate experiments.

\subsection{Immobilization of TTAS}

In order to isolate and identify the intracellular target of TTAS in Leishmania infantum we decided to immobilize the compound and to isolate its target(s) by an affinity chromatography approach. The SAR based on the $\mathrm{LD}_{50}$ values (Table 1 ) suggests that the addition of a hydrogen bond donor/acceptor in meta position (4 vs $\mathbf{3}$ ) of the phenyl moiety is not accompanied by a dramatic amelioration of the activity. Thus, it has been hypothesized that the stilbene scaffold may represent the pharmacophore responsible for the activity. In line with this hypothesis, TTAS has been chosen for performing the affinity chromatography because it guarantees a unique binding to the matrix via the primary amino group which react more easily with the terminal epoxy group of the activated agarose then hydroxyl groups (as in compounds 5), providing an uncharged, hydrophilic and very stable 12 -atoms spacer (Fig. 6). Direct absorbance scans of the immobilized ligand on the matrix resuspended in $50 \%$ glycerol solution $(\mathrm{v} / \mathrm{v})$ confirmed successful coupling (data not shown). Routinely $50-80 \mu \mathrm{mol} / \mathrm{mL}$ were bound.

\section{6. $\beta$-tubulin binds to the immobilized compound TTAS}

The total parasite lysate was prepared, loaded into the matrices and unbound material was removed as outlined in Section 2 . The bound proteins were separated by SDS-PAGE and visualized by silver-staining (Fig. 7). The compound TTAS-derived matrix led to the detection of three distinct protein bands, two of which at higher molecular weights also being present in the control (Fig. 7). Trypsin digestion and peptide sequencing by LC/ESI/MS/MS-QTOF mass spectrometry identified the specific band at $\sim 49 \mathrm{kDa}$ as $\beta$-tubulin of Leishmania infantum (expected size of $49.7 \mathrm{kDa}$; score 82 ), whereas the other two correspondend to human keratin (scores 168 and 65). Human keratin that was present in both the control matrix and the TTAS-derived matrix was the predominant contamination which turned out to be present under any circumstances, and it was even found in new batches of matrix that were stored in sealed containers before first use.

\section{Discussion}

Leishmaniasis treatment is based on parenteral administration of highly toxic drugs including pentavalent antimonials, amphotericin B in its liposomal formulation (Ambisone) and pentamidine (Murray et al., 2005; Croft et al., 2006; Baiocco et al., 2009; Palumbo, 2009). Recently, oral administration of miltefosine has emerged as an alternative approach (Herwaldt, 1999). It was initially identified as an anticancer drug and its antiprotozoal activity was subsequently demonstrated against visceral and cutaneous leishmaniasis. Unfortunately, despite its great efficacy, miltefosine is limited by its extremely long half-life, and low therapeutic index.

In view of the foregoing facts, there is an urgent need for the development of antileishmania agents based on new molecular scaffold endowed with improved efficacy and lacking toxicity.

Recently, trans-resveratrol, a natural stilbene compound, has been studied for its leishmanicidal properties. Resveratrol has 

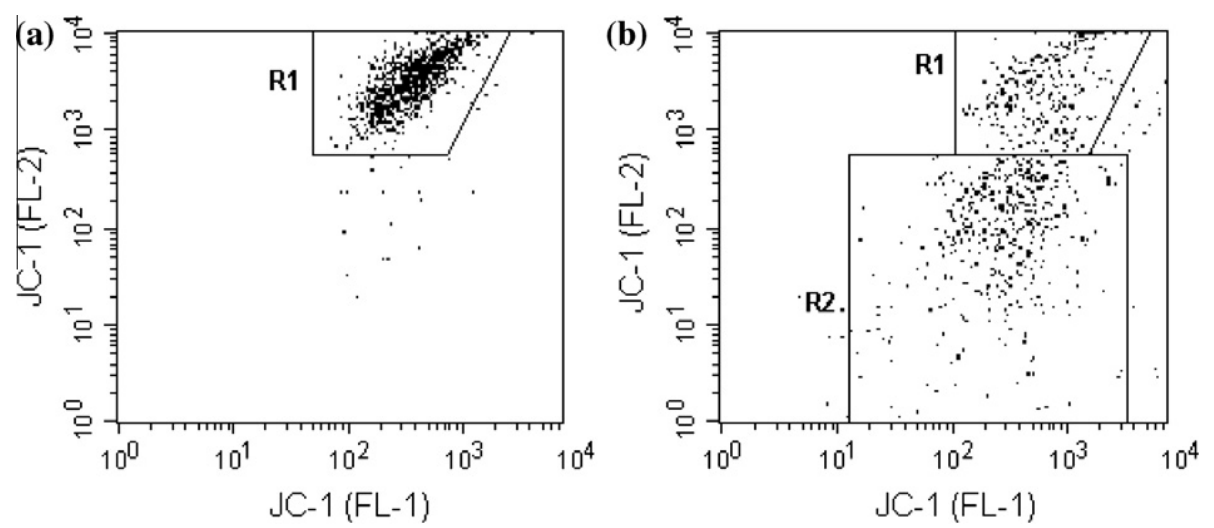

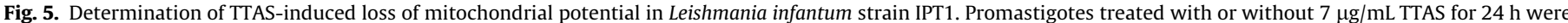

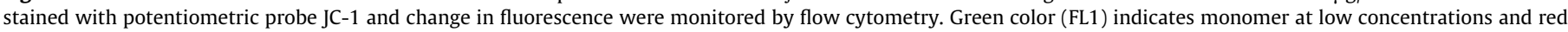

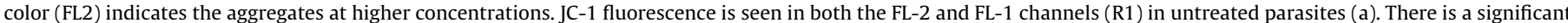

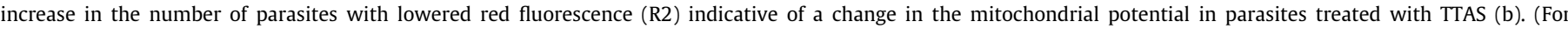
interpretation of the references to colour in this figure legend, the reader is referred to the web version of this article).<smiles>COc1cc(/C=C/c2ccc(OC)c(N)c2)cc(OC)c1</smiles><smiles>COc1cc(/C=C/c2ccc(NCC(O)COCCCCOCC(O)COc3ccccc3)c(OC)c2)cc(OC)c1</smiles>

Fig. 6. Affinity matrix preparation. Trans-3,4',5-trimethoxy-3'-amino-stilbene 6 (TTAS) was coupled to epoxy-activated agarose consisting of a 12-atoms spacer (1,4-bis(2:3epoxypropoxy)butane) to form affinity matrix. The agarose beads are shown as spheres.

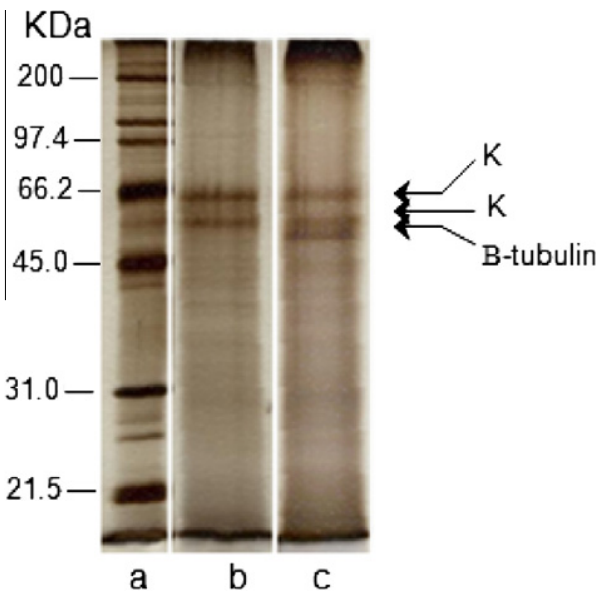

Fig. 7. SDS-PAGE (12\%) analysis of proteins reteined by the affinity matrix. Molecular mass marker (lane a). Samples of Leishmania infantum lysate $(120 \mu \mathrm{g}$ total protein content) were incubated with control beads (lane b), or affinity beads (lane c). Proteins retained by the matrices were directly separated by SDS-PAGE. Arrows indicate protein bands resulting from the compound derived matrices. K: human keratin. shown antileishmanial activity when tested in vitro on Leishmania major promastigotes (Kedzierski et al., 2007). Microscopic examination indicated that the effect was leishmanicidal rather than leishmanistatic, as promastigote morphology indicated cells were dead, rather than their growth was slowed down. This is interesting considering that resveratrol is well-tolerated in vivo and it is no toxic at doses 1000 times higher than a normal daily intake (Juan et al., 2002). To obtain compounds endowed with higher antileishmanial activity, new resveratrol derivatives have been synthesized and tested on Leishmania parasites and normal human cells. However, most of them have shown a toxicity on normal fibroblasts and myeloid cells similar or higher than that observed in Leishmania parasites (Kedzierski et al., 2007).

Previously we have described the synthesis of new cis- and trans-resveratrol based derivatives with the aim of discovering new lead compounds with anticancer potential (Roberti et al., 2003; Tolomeo et al., 2005). In this work we investigated the anti-leishmania activity of some of them, with the aim of discovering new lead candidates or tools for the discovery of new therapeutically relevant targets.

Therefore stilbene derivatives 1-7 were assayed for their ability to inhibit the growth of Leishmania infantum promastigotes and amastigotes in vitro. All the compounds were capable to decrease 
the parasite viability in a dose-dependent manner. As shown in Table 1 , the cis-stilbene derivatives $\mathbf{1}, \mathbf{2}$ and $\mathbf{7}$ showed an $\mathrm{LD}_{50}$ higher than their correspondent trans-isomers 4, 5 and 6 both in promastigotes and amastigotes. This was somewhat surprising to us because in our previous studies on leukemia cells, the cis-stilbene derivatives showed higher cytotoxic and pro-apoptotic activity than the parent trans-isomers (Roberti et al., 2003). The activity of pterostilbene $3\left(\mathrm{LD}_{50}\right.$ of $\left.7.6 \mu \mathrm{g} / \mathrm{mL}\right)$ was comparable to that of its 3'-hydroxyl derivative 4 suggesting that the introduction of the second hydroxyl function in meta position is not influencing the leishmanicidal activity. Once again the result differed from that previously obtained on leukemia cells where the above modification resulted in about 40 -fold increase in both cytotoxic and proapoptotic activity. In the present study the most active compound was trans-3,4',5-trimethoxy-3'-amino-stilbene (6, TTAS) that showed a $\mathrm{LD}_{50}$ of $2.6 \mu \mathrm{g} / \mathrm{mL}$. Of interest, this compound showed an $\mathrm{IC}_{50}$ on normal CFU-GM of $17.7 \mu \mathrm{g} / \mathrm{mL}$, therefore more than 6 times higher than that showed on the Leishmania infantum strain. For this selectivity TTAS may be considered an interesting compound for the antileishmanial therapy development, therefore we tried to understand its antileishmanial mechanism of action. Leishmania parasites treated $24 \mathrm{~h}$ with TTAS were blocked prevalently in $\mathrm{G}_{2}-\mathrm{M}$ phase of cell cycle and after $48 \mathrm{~h}$ of TTAS exposure Leishmania parasites were induced in apoptosis as shown by morphologic examination and Annexin $\mathrm{V}$ positivity. The loss of mitochondrial membrane potential suggests that the TTAS-induced apoptosis is mediated by the mitochondrial apoptotic pathway. To discover the main molecular target/s of TTAS in Leishmania parasites we took advantage of a chemical proteomic approach that was recently successfully applied by some of us (Kuettel et al., 2009). Thus the amino-stilbene derivative TTAS, chosen based on the presented activity data, was immobilized and the corresponding affinity matrix was used to find out potential targets. Indeed this approach identified $\beta$-tubulin as putative specific target of TTAS in Leishmania consistently with the ability of TTAS to block Leishmania parasites in $\mathrm{G}_{2}-\mathrm{M}$ phase of cell cycle.

\section{Acknowledgment}

We are grateful to Dr. Remo Perozzo and Dr. Karl Werbovetz for the fruitful discussion. We thank the University of Bologna for the financial support.

\section{References}

Baiocco, P., Colotti, G., Franceschini, S., Ilari, A., 2009. Molecular basis of antimony treatment in leishmaniasis. J. Med. Chem. 52, 2603-2612.

Cao, T.M., Durrant, D., Tripathi, A., Liu, J., Tsai, S., Kellogg, G.E., Simoni, D., Lee, R.M., 2008. Stilbene derivatives that are colchicine-site microtubule inhibitors have antileukemic activity and minimal systemic toxicity. Am. J. Hematol. 83, 390397.

Changtam, C., de Koning, H.P., Ibrahim, H., Sajid, M.S., Gould, M.K., Suksamrarn, A., 2010. Curcuminoid analogs with potent activity against Trypanosoma and Leishmania species. Eur. J. Med. Chem. 45, 941-956.

Colomba, C., Saporito, L., Vitale, F., Reale, S., Vitale, G., Casuccio, A., Tolomeo, M., Maranto, D., Rubino, R., Di Carlo, P., Titone, L., 2009. Cryptic Leishmania infantum infection in Italian HIV infected patients. BMC Infect. Dis. 9, 199.

Croft, S.L., Seifert, K., Yardley, V., 2006. Current scenario of drug development for leishmaniasis. Indian J. Med. Res. 123, 399-410.

Cruz, I., Nieto, J., Moreno, J., Canavate, C., Desjeux, P., Alvar, J., 2006. Leishmania/HIV co-infections in the second decade. Indian J. Med. Res. 123, 357-388. del Olmo, E., Armas, M.G., Lopez-Perez, J.L., Munoz, V., Deharo, E., San Feliciano, A., 2001. Leishmanicidal activity of some stilbenoids and related heterocyclic compounds. Bioorg. Med. Chem. Lett. 11, 2123-2126.

del Rey, B., Ramos, A.C., Caballero, E., Inchaustti, A., Yaluff, G., Medarde, M., Rojas de Arias, A., San Feliciano, A., 1999. Leishmanicidal activity of combretastatin analogues and heteroanalogues. Bioorg. Med. Chem. Lett. 9, 2711-2714.

Duarte, N., Kayser, O., Abreu, P., Ferreira, M.J., 2008. Antileishmanial activity of piceatannol isolated from Euphorbia lagascae seeds. Phytother. Res. 22, 455457.

Fremont, L., 2000. Biological effects of resveratrol. Life Sci. 66, 663-673.

Getti, G.T., Aslam, S.N., Humber, D.P., Stevenson, P.C., Cheke, R.A., 2006. The effect of cicerfuran, an arylbenzofuran from Cicer bijugum, and related benzofurans and stilbenes on Leishmania aethiopica, L. tropica and L. major. Planta Med. 72, 907911

Hart, J.H., 1981. Role of phytostilbenes in decay and disease resistance. Ann. Rev. Phytopathol. 19, 437-458.

Herwaldt, B.L., 1999. Miltefosine-the long-awaited therapy for visceral leishmaniasis? N. Engl. J. Med. 341, 1840-1842.

Juan, M.E., Vinardell, M.P., Planas, J.M., 2002. The daily oral administration of high doses of trans-resveratrol to rats for 28 days is not harmful. J. Nutr. 132, 257260.

Kedzierski, L., Curtis, J.M., Kaminska, M., Jodynis-Liebert, J., Murias, M., 2007. In vitro antileishmanial activity of resveratrol and its hydroxylated analogues against Leishmania major promastigotes and amastigotes. Parasitol. Res. 102, 91-97.

Kuettel, S., Mosimann, M., Maser, P., Kaiser, M., Brun, R., Scapozza, L., Perozz, R., 2009. Adenosine Kinase of $\mathrm{T} \mathrm{b}$. Rhodesiense identified as the putative target of 4-[5-(4-phenoxyphenyl)-2H-pyrazol-3-yl] morpholine using chemical proteomics. PLoS Negl. Trop. Dis. 3, e506.

Marin, C., Boutaleb-Charki, S., Diaz, J.G., Huertas, O., Rosales, M., Perez-Cordon, G. Guitierrez-Sanchez, R., Sanchez-Moreno, M., 2009. Antileishmaniasis activity of flavonoids from Consolida oliveriana. J. Nat. Prod. 72, 1069-1074.

Mishra, B.B., Kale, R.R., Singh, R.K., Tiwari, V.K., 2009a. Alkaloids: future prospective to combat leishmaniasis. Fitoterapia 80, 81-90.

Mishra, B.B., Singh, R.K., Srivastava, A., Tripathi, V.J., Tiwari, V.K., 2009b. Fighting against Leishmaniasis: search of alkaloids as future true potential antiLeishmanial agents. Mini Rev. Med. Chem. 9, 107-123.

Murray, H.W., Berman, J.D., Davies, C.R., Saravia, N.G., 2005. Advances in leishmaniasis. Lancet 366, 1561-1577.

Palumbo, E., 2009. Current treatment for cutaneous leishmaniasis: a review. Am. J. $16,178-182$.

Roberti, M., Pizzirani, D., Simoni, D., Rondanin, R., Baruchello, R., Bonora, C., Buscemi, F., Grimaudo, S., Tolomeo, M., 2003. Synthesis and biological evaluation of resveratrol and analogues as apoptosis-inducing agents. J. Med. Chem. 46, 3546-3554.

Sen, N., Das, B.B., Ganguly, A., Mukherjee, T., Bandyopadhyay, S., Majumder, H.K., 2004a. Camptothecin-induced imbalance in intracellular cation homeostasis regulates programmed cell death in unicellular hemoflagellate Leishmania donovani. J. Biol. Chem. 279, 52366-52375.

Sen, N., Das, B.B., Ganguly, A., Mukherjee, T., Tripathi, G., Bandyopadhyay, S., Rakshit, S., Sen, T., Majumder, H.K., 2004b. Camptothecin induced mitochondrial dysfunction leading to programmed cell death in unicellular hemoflagellate Leishmania donovani. Cell Death Differ. 11, 924-936.

Sengupta, S., Tripathi, J., Tandon, R., Raje, M., Roy, R.P., Basu, S.K., Mukhopadhyay, A., 1999. Hemoglobin endocytosis in Leishmania is mediated through a 46-kDa protein located in the flagellar pocket. J. Biol. Chem. 274, 2758-2765.

Shen, T., Wang, X.N., Lou, H.X., 2009. Natural stilbenes: an overview. Nat. Prod. Rep. 26, 916-935.

Singh, G., Jayanarayan, K.G., Dey, C.S., 2005. Novobiocin induces apoptosis-like cell death in topoisomerase II over-expressing arsenite resistant Leishmania donovani. Mol. Biochem. Parasitol. 141, 57-69.

Susin, S.A., Zamzami, N., Kroemer, G., 1998. Mitochondria as regulators of apoptosis: doubt no more. Biochim. Biophys. Acta 1366, 151-165.

Tipparaju, S.K., Joyasawal, S., Pieroni, M., Kaiser, M., Brun, R., Kozikowski, A.P., 2008. In pursuit of natural product leads: synthesis and biological evaluation of 2-[3hydroxy-2-[(3-hydroxypyridine-2-carbonyl)aminolphenyl]benzoxazole-4-c arboxylic acid (A-33853) and its analogues: discovery of N-(2-benzoxazol-2ylphenyl)benzamides as novel antileishmanial chemotypes. J. Med. Chem. 51, 7344-7347.

Tolomeo, M., Grimaud, S., Di Cristina, A., Roberti, M., Pizzirani, D., Meli, M., Dusonchet, L., Gebbia, N., Abbadessa, V., Crosta, L., Barucchello, R., Grisolia, G., Invidiata, F., Simoni, D., 2005. Pterostilbene and 3'-hydroxypterostilbene are effective apoptosis-inducing agents in MDR and BCR-ABL-expressing leukemia cells. Int. J. Biochem. Cell Biol. 37, 1709-1726.

Young, S.L., Chaplin, D.J., 2004. Combretastatin A4 phosphate: background and current clinical status. Expert Opin. Investig. Drugs 13, 1171-1182. 